\title{
Relational Representation of Groupoid Quantales
}

\author{
Alessandra Palmigiano • Riccardo Re
}

Received: 8 May 2011 / Accepted: 11 July 2011 / Published online: 29 July 2011

(C) The Author(s) 2011. This article is published with open access at Springerlink.com

\begin{abstract}
In Palmigiano and Re (J Pure Appl Algebra 215(8):1945-1957, 2011), spatial SGF-quantales are axiomatically introduced and proved to be representable as sub unital involutive quantales of quantales arising from set groupoids. In the present paper, spatial SGF-quantales of this class are shown to be optimally representable as unital involutive quantales of relations. The results of the present paper have several aspects in common with Jónsson and Tarski's representation theory for relation algebras (Jónsson and Tarski, Am J Math 74(2):127-162, 1952).
\end{abstract}

Keywords Unital involutive quantale • Strongly Gelfand quantale • Set groupoid • Representation theorem

Mathematics Subject Classifications (2010) 06D05 - 06D22 - 06D50 • 06F07 • 18B40 • $20 \mathrm{~L} 05 \cdot 22 \mathrm{~A} 22 \cdot 54 \mathrm{D} 10 \cdot 54 \mathrm{D} 30 \cdot 54 \mathrm{D} 80$

\section{Introduction}

Quantales were introduced [6] as noncommutative generalizations of locales, and have been investigated in close connection with groupoids, e.g. in [7-9, 11, 13]. In particular, in [8] a certain class of unital involutive quantales, called spatial

The research of the first author was made possible by the VENI grant 639.031.726 of the Netherlands Organization for Scientific Research (NWO).

\footnotetext{
A. Palmigiano $(\bowtie)$

Institute for Logic, Language and Computation, Universiteit van Amsterdam, P.O. Box 94242, 1090 GE Amsterdam, The Netherlands

e-mail: a.palmigiano@uva.nl

R. Re

Dipartimento di Matematica e Informatica, Università di Catania,

Viale Andrea Doria, 6, 95125 Catania, Italy

e-mail: riccardo@dmi.unict.it
} 
$S G F$-quantales, is axiomatically introduced and proved to bijectively correspond to structures based on groupoids. Key to this result is the proof that spatial SGFquantales are representable as sub unital involutive quantales of quantales arising from set groupoids.

The present paper was motivated by the question whether an optimal relational representation could be given for spatial SGF-quantales, i.e., whether any spatial SGF-quantale could be embedded into the powerset of some equivalence relation in such a way that joins are represented as unions, the product as standard relational composition, the product unit as the identity relation, and the quantale involution as relational converse. Relational representability for quantales has already been studied by some authors in the literature: for instance, in [1] it is shown that every quantale $\mathcal{Q}$ can be order-embedded into a quantale of relations in such a way that the noncommutative product is represented as relational composition, but this method does not extend to involutive quantales and the join of $\mathcal{Q}$ is not in general represented as the union. Similar limitations apply to the results in [16]. In [4], it is shown that any involutive quantale is embeddable into a quantale of join-semilattice endomorphisms, which give back the quantales of relations when the join-semilattice is a powerset.

Quantales and relation algebras are akin: it is well known that both kinds of algebras naturally arise from groupoids. Concrete relation algebras have been identified by Jónsson and Tarski as those embeddable into relation algebras arising from groupoids (cf. [3, Theorem 5.8]). In the light of these facts, the present paper was set out to extend the optimal representation theory of relation algebras, as is developed in [3], to spatial SGF-quantales. The Jónsson-Tarski representation theory is grounded in the theory of canonical extensions. In particular, it is crucial that any relation algebra can be canonically embedded into its perfect, or canonical extension [3, Theorem 4.21], which is an atomistic relation algebra (i.e. it is joingenerated by its atoms). In the present paper, the role of atomistic relation algebras is played by FA-quantales (Definition 4.1), and the role of the canonical embedding is played by the map $\alpha$ in Theorem 3.5.

Before moving on, it is worth mentioning ${ }^{1}$ that, besides the similarities between quantales and relation algebras, on which the present paper is based, similarities exist between the embedding of Corollary 4.15 and the Gelfand-Naimark embedding [2] of any $C^{*}$-algebra $A$ into the algebra of bounded operators $\mathcal{B}(\mathcal{H})$ of some Hilbert space $\mathcal{H}$. For the sake of this analogy, the role of $A$ is played by any SGF-quantale $Q$, which is associated with some groupoid $\mathcal{G}(\mathcal{Q})=\left(G_{1}, G_{0}\right)$, and the role of the von Neumann algebra $\mathcal{B}(\mathcal{H})$ is played by the quantale $\mathcal{P}\left(G_{1}\right)$, into which $Q$ is embedded (cf. Corollary 4.15). This analogy can be even pushed further: indeed, the role that the pure states of the $C^{*}$ algebra play in the Gelfand-Naimark embedding via the Gelfand-Naimark-Segal construction is similar to the role played by the equivalence classes $[p, f]$ defined right before Definition 3.3 in the present paper. Moreover, the FA-quantales into which SGF-quantales embed, which are used in the present paper as intermediate structures to obtain the representation theorem, can be regarded as the quantale-theoretic counterparts of the enveloping von Neumann algebras of any $C^{*}$-algebra $A$.

${ }^{1}$ We wish to thank the anonymous referee for bringing this to our attention. 
Finally, Proposition 4.17 provides a characterization of quantales of the form $\mathcal{P}(R)$ for some equivalence relation $R$ on some set $X$. Any such quantale can be identified with the cartesian product of the discrete pair quantales $\mathcal{P}\left(X_{i} \times X_{i}\right)$, where $\left\{X_{i}: i \in I\right\}$ is the partition of $X$ induced by $R$; in the light of this observation, the characterization of Proposition 4.17 establishes a similarity between quantales of the form $\mathcal{P}(R)$ and von Neumann algebras which decompose as direct integrals of type I factors (cf. [15]). Summing up, relative to the quantales and the $C^{*}$-algebras bijectively corresponding to groupoids, the following list of systematic analogies emerges:

\begin{tabular}{|c|c|}
\hline (Groupoid) $C^{*}$-algebras & (Groupoid) quantales \\
\hline $\begin{array}{r}C^{*} \text {-algebras } \\
\text { von Neumann algebras } \\
\text { direct integrals of type I factors } \\
\text { type I factors }\end{array}$ & $\begin{array}{l}\text { SGF-quantales } \mathcal{Q} \\
\text { discrete groupoid quantales } \mathcal{P}(\mathcal{G}) \\
\text { relational quantales } \mathcal{P}(R) \\
\text { discrete pair quantales } \mathcal{P}(X \times X)\end{array}$ \\
\hline
\end{tabular}

Developing these analogies further will be the object of future work, as well as the question whether quantale-theoretic counterparts exist to type II and III factors in the theory of von Neumann algebras.

The paper is organized as follows: in Section 2 we give preliminaries on unital involutive quantales and groupoids. In Section 3 we report on the relevant definitions and results from [8], up to Theorem 3.5. Section 4 collects the original contributions of the paper: in Section 4.1, we develop the theory of FA-quantales, and show that they are identifiable with the quantales $\mathcal{P}(\mathcal{G})$ for any groupoid $\mathcal{G}$ (Proposition 4.6); in Section 4.2 we provide a dual characterization of homomorphisms and embeddings between FA-quantales (Propositions 4.7, 4.9, and 4.10). In Sections 4.3 and 4.4, we give two applications of this dual characterization: the first one (Corollary 4.15) is an optimal representation theorem for spatial SGF-quantales as sub unital involutive quantales of quantales of relations; this result is the quantale-theoretic counterpart of Theorem 4.22 in [3]. The second one (Theorem 4.21) is a characterization of those unital involutive quantales which are isomorphic to quantales of the form $\mathcal{P}(R)$ for some equivalence relation $R$; this result is the quantale-theoretic counterpart of Theorem 4.30 in [3].

\section{Preliminaries}

\subsection{Strongly Gelfand Quantales}

A quantale $\mathcal{Q}[6,14]$ is a complete join-semilattice endowed with an associative binary operation - that is completely distributive in each coordinate, i.e.:

$\mathrm{D} 1: \quad c \cdot \bigvee I=\bigvee\{c \cdot q: q \in I\}$

D2: $\bigvee I \cdot c=\bigvee\{q \cdot c: q \in I\}$

for every $c \in \mathcal{Q}$ and every $I \subseteq \mathcal{Q}$. Since it is a complete join-semilattice, any quantale $\mathcal{Q}$ is also a complete, hence bounded, lattice. Let 0,1 be the lattice bottom and top of $\mathcal{Q}$, respectively. Conditions D1 and D2 readily imply that · is order-preserving in 
both coordinates and, as $\bigvee \varnothing=0$, that $c \cdot 0=0=0 \cdot c$ for every $c \in \mathcal{Q}$. A quantale $\mathcal{Q}$ is unital if there exists some element $e \in \mathcal{Q}$ for which

$\mathrm{U}: \quad e \cdot c=c=c \cdot e$ for every $c \in \mathcal{Q}$,

and is involutive if it is endowed with a unary operation $*$ such that, for all $c, q \in \mathcal{Q}$ and every $I \subseteq \mathcal{Q}$,

I1: $\quad c^{* *}=c$;

I2: $(c \cdot q)^{*}=q^{*} \cdot c^{*}$;

I3: $(\bigvee I)^{*}=\bigvee\left\{q^{*}: q \in I\right\}$.

Examples of unital involutive quantales, which will turn out to arise from groupoids, are:

1. The quantale $\mathcal{P}(R)$ of subrelations of a given equivalence relation $R \subseteq X \times X$; the two projections define the maps $d$ and $r$, composition is given by $(x, y)$. $(y, z)=(x, z)$, the diagonal map $x \mapsto(x, x)$ gives the unit $u$, and $i(x, y):=(y, x)$.

2. The quantale $\mathcal{P}(G)$, for every group $G$. The structure maps are canonically given by the algebraic structure of $G$.

3. Any frame $\mathcal{Q}$, setting $:=\wedge, *:=\mathrm{id}$ and $e:=1_{\mathcal{Q}}$.

A homomorphism of (involutive) quantales is a map $\varphi: \mathcal{Q} \rightarrow \mathcal{Q}^{\prime}$ that preserves $\bigvee$, . (and $*$ ). If $\mathcal{Q}$ and $\mathcal{Q}^{\prime}$ are unital quantales, then $\varphi$ is unital if $e^{\prime} \leq \varphi(e)$ and is strictly unital if $\varphi(e)=e^{\prime}$. Notice that since every homomorphism is completely join-preserving, then $\varphi(0)=\varphi(\bigvee \varnothing)=\bigvee \varnothing=0$. However, a homomorphism of quantales does not need to preserve the lattice top. For example, if $R \varsubsetneqq S$ are equivalence relations on $X$, then the inclusion $\mathcal{P}(R) \rightarrow \mathcal{P}(S)$ is a strictly unital homomorphism of quantales that does not preserve the lattice top. If $\varphi\left(1_{\mathcal{Q}}\right)=1_{\mathcal{Q}^{\prime}}$ then $\varphi$ is strong.

Let $\mathcal{Q}$ be a unital involutive quantale. An element $f \in \mathcal{Q}$ is functional if $f^{*} \cdot f \leq e$ and is a partial unit if both $f$ and $f^{*}$ are functional. ${ }^{2}$ The set of functional elements (resp. partial units) will be denoted by $\mathcal{F}(\mathcal{Q})$ (resp. $\mathcal{I}(\mathcal{Q})$ ). It is easy to verify that $e \in \mathcal{I}(\mathcal{Q})$ and $\mathcal{I}(\mathcal{Q})$ is closed under the composition and involution of $\mathcal{Q}$. Moreover, if $f \leq g \in \mathcal{I}(\mathcal{Q})$ then $f \in \mathcal{I}(\mathcal{Q})$.

Let $\mathcal{Q}_{e}=\{c \in \mathcal{Q}: c \leq e\}$. Then $\mathcal{Q}_{e} \subseteq \mathcal{I}(\mathcal{Q})$; moreover, $\mathcal{Q}_{e}$ is a unital involutive subquantale of $\mathcal{Q}$.

Definition 2.1 A unital involutive quantale $\mathcal{Q}$ is strongly Gelfand (or an $S G$ quantale) if

SG. $a \leq a \cdot a^{*} \cdot a$ for every $a \in \mathcal{Q}$.

Recall that $\mathcal{Q}$ is a Gelfand quantale (see also [14]) if $a=a \cdot a^{*} \cdot a$ for every rightsided element of $\mathcal{Q}$ ( $a \in \mathcal{Q}$ being right-sided if $a=a \cdot 1)$. It is immediate to see that every SG-quantale is Gelfand, and that $f=f \cdot f^{*} \cdot f$ for every SG-quantale $\mathcal{Q}$ and every $f \in \mathcal{F}(\mathcal{Q})$. We will simplify notation and write $a \cdot b$ as $a b$.

${ }^{2}$ If $\mathcal{Q}=\mathcal{P}(R)$ for some equivalence relation $R \subseteq X \times X$, then functional elements (partial units) are exactly the graphs of (invertible) partial maps $f$ on $X$. 


\section{Proposition 2.2 For every $S G$-quantale $\mathcal{Q}$,}

1. the subquantale $\mathcal{Q}_{e}$ is a frame: in particular, involution $*$ coincides with the identity, and composition $\cdot$ with $\wedge$.

2. $\mathcal{I}(\mathcal{Q})$ is an inverse monoid ${ }^{3}$ whose set of idempotents coincides with $\mathcal{Q}_{e}$, and whose natural order coincides with the order inherited from $\mathcal{Q}$.

Proof

1. Let $d \leq e$. By SG, we have that $d \leq d d^{*} d \leq e d^{*} e=d^{*}$, and likewise, $d^{*} \leq d$, hence involution coincides with the identity on $\mathcal{Q}_{e}$. If $c \leq e$, then $c c=c$ : indeed, $c c \leq c e=c$, and by SG and the fact that involution is identity on $\mathcal{Q}_{e}$, we get that $c=c c^{*} c=(c c) c \leq(c c) e=c c$. Let $d_{1}, d_{2} \leq e$. Then $d_{1} d_{2} \leq d_{1} e=d_{1}$ and $d_{1} d_{2} \leq$ $e d_{2}=d_{2}$, so $d_{1} d_{2} \leq d_{1} \wedge d_{2}$. Conversely, if $c \leq d_{1}$ and $c \leq d_{2}$, then $c=c c \leq d_{1} d_{2}$, hence $d_{1} \wedge d_{2} \leq d_{1} d_{2}$.

2. By SG, we get that $f f^{*} f=f$ and $f^{*} f f^{*}=f^{*}$ for every $f \in \mathcal{I}(\mathcal{Q})$. Hence, it is enough to show that the restriction of the product to the idempotent elements of $\mathcal{I}(\mathcal{Q})$ is commutative. This follows from item 1 above and from the fact that for every $f \in \mathcal{I}(\mathcal{Q})$, it holds that $f f=f$ iff $f \leq e$ : Indeed, if $f \leq e$, then by (1), we have that $f f=f \wedge f=f$. Conversely, if $f f=f$, then $f^{*}=(f f)^{*}=f^{*} f^{*}$, hence $f f^{*}=f f^{*} f^{*} \leq e f^{*}=f^{*}$, and so $f=f f^{*} f \leq f^{*} f \leq e$. Since $\mathcal{Q}_{e} \subseteq \mathcal{I}(\mathcal{Q})$, this also shows that the set of idempotent elements of $\mathcal{I}(\mathcal{Q})$ coincides with $\mathcal{Q}_{e}$. Hence, the natural order of the inverse monoid $\mathcal{I}(\mathcal{Q})$ is defined as follows: $f \leq g$ iff $f=g h$ for some $h \in \mathcal{Q}_{e}$, and therefore it coincides with the order inherited from $\mathcal{Q}$.

\subsection{Groupoids}

Definition 2.3 A set groupoid is a tuple $\mathcal{G}=\left(G_{0}, G_{1}, m, d, r, u, i\right)$, s.t.:

G1. $G_{0}$ and $G_{1}$ are sets;

G2. $d, r: G_{1} \rightarrow G_{0}$ and $u: G_{0} \rightarrow G_{1}$ s.t. $d(u(p))=p=r(u(p))$ for every $p \in G_{0}$;

G3. $m:(x, y) \mapsto x y$ is an associative map defined on $G_{1} \times_{G_{0}} G_{1}=\{(x, y) \mid r(x)=$ $d(y)\}$ and s.t. $d(x y)=d(x)$ and $r(x y)=r(y)$;

G4. $x u(r(x)))=x=u(d(x)) x$ for every $x \in G_{1}$;

G5. the map $i: G_{1} \rightarrow G_{1}$ denoted by $i(x)=x^{-1}$ is s.t. $x x^{-1}=u(d(x)), x^{-1} x=$ $u(r(x)), d\left(x^{-1}\right)=r(x)$ and $r\left(x^{-1}\right)=d(x)$ for every $x \in G_{1}$.

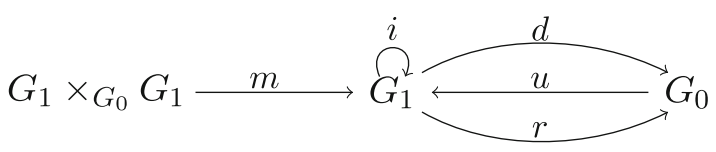

Axioms G1-5 equivalently say that groupoids are small categories ( $G_{0}$ being the set of objects, $G_{1}$ being the set of arrows, $u$ being the injective map which identifies

\footnotetext{
${ }^{3}$ An inverse semigroup (cf. [10]) is a semigroup such that for every element $x$ there exists a unique inverse, i.e. an element $y$ such that $x=x y x$ and $y=y x y$. Equivalently, an inverse semigroup is a semigroup such that every element has some inverse and any two idempotent elements commute. An inverse monoid is an inverse semigroup with a multiplicative unit. The natural order on an inverse semigroup is defined by $x \leq y$ iff $x=e \cdot y$ for some idempotent $e$.
} 
any object with its identity arrow, etc) such that every arrow is an iso. Then a morphism of groupoids $f: \mathcal{G} \rightarrow \mathcal{G}^{\prime}$ ( $\mathcal{G}^{\prime}$ has structure maps $d^{\prime}, r^{\prime}$, etc) is a functor from $\mathcal{G}$ to $\mathcal{G}^{\prime}$. In other words, a morphism $f$ consists of a pair of functions $f_{1}: G_{1} \rightarrow G_{1}^{\prime}$ and $f_{0}: G_{0} \rightarrow G_{0}^{\prime}$ that preserve the structure maps of the groupoids in the appropriate way; that is, for every $p \in G_{0}$ and all $x, y \in G_{1}$ :

$$
\begin{array}{ll}
f_{0}(d(x))=d^{\prime}\left(f_{1}(x)\right), & f_{0}(r(x))=r^{\prime}\left(f_{1}(x)\right), \\
f_{1}(u(p))=u^{\prime}\left(f_{0}(p)\right), & f_{1}(x y)=f_{1}(x) f_{1}(y) .
\end{array}
$$

Clearly, the map $f_{0}$ is completely determined by $f_{1}$, so $f$ can be identified with $f_{1}$. Groupoid morphisms preserve inverses: $f\left(x^{-1}\right)=f(x)^{-1}$ (to see this, use Lemma 2.5.4 below). An isomorphism of groupoids $f: \mathcal{G} \rightarrow \mathcal{G}^{\prime}$ is a morphism which has an inverse functor $f^{-1}: \mathcal{G}^{\prime} \rightarrow \mathcal{G}$. The groupoids $\mathcal{G}$ and $\mathcal{G}^{\prime}$ are isomorphic if there is an isomorphism $f: \mathcal{G} \rightarrow \mathcal{G}^{\prime}$.

\section{Example 2.4}

1. For any equivalence relation $R \subseteq X \times X$, the tuple $\left(X, R, \circ, \pi_{1}, \pi_{2}, \Delta,()^{-1}\right)$ defines a groupoid. Conversely, for every groupoid $\mathcal{G}$, if the product map $d \times r: G_{1} \rightarrow$ $G_{0} \times G_{0}$ is injective, then $\mathcal{G}$ is isomorphic to the groupoid arising from the equivalence relation $(d \times r)\left[G_{1}\right] \subseteq G_{0} \times G_{0}$ (cf. [12], Example I.1.3). Groupoids arising from the relation $R=X \times X$ for some set $X$ are referred to as pair groupoids.

2. For any group $\left(G, \cdot, e,()^{-1}\right)$, the tuple $\left(\{e\}, G, \cdot, d, r, u,()^{-1}\right)$ is a groupoid, and the equalities G4 and G5 just restate the group axioms.

3. Every topological space $X$ can be seen as a groupoid by setting $G_{1}=G_{0}=X$ and identity structure maps. In this case, $G_{1} \times_{G_{0}} G_{1}=\{(x, x) \mid x \in X\}$ and $x x=x$ for every $x \in X$.

4. A groupoid can be associated with any action ${ }^{4} G \times X \rightarrow X$ of a group $G$ on a set $X$, by setting $G_{1}=G \times X, G_{0}=X$, and for all $g, h \in G$ and $x, y \in X$, $d(g, x)=x, r(g, x)=g x, u(x)=(e, x)(e \in G$ being the identity element $)$, and $(g, x) \cdot(h, y)=(h g, x)$ whenever $y=g x$.

5. To a group action as above, another groupoid can be associated, which is given by the equivalence relation $R \subseteq X \times X$ defined by $x R y$ iff there exists some $g \in G$ such that $y=g x$.

Some useful well known facts about groupoids are reported in the following:

Lemma 2.5 For every $p \in G_{0}$, and all $x, y \in G_{1}$,

1. $u(p)^{-1}=u(p)$,

2. $x=x x^{-1} x$ and $x^{-1}=x^{-1} x x^{-1}$,

3. if $x y^{-1}, x^{-1} y \in u\left[G_{0}\right]$ then $x=y$,

4. if $x=x y x$ and $y x y=y$, then $y=x^{-1}$,

5. $\left(x^{-1}\right)^{-1}=x$,

6. $(x y)^{-1}=y^{-1} x^{-1}$.

\footnotetext{
${ }^{4}$ For any group $G$, a (left) action of $G$ on a set $X$ is a function $\cdot: G \times X \rightarrow X$ s.t. for all $g, h \in G$ and every $x \in X$, the identities $(g h) x=g(h x)$ and $e x=x$ hold ( $e$ being the identity of $G$ ). For any topological group $G$ and any topological space $X$, a continuous action of $G$ on $X$ is a continuous map $G \times X \rightarrow X$ which is an action of $G$ as a discrete group $G$ on the underlying set of $X$.
} 
Proof The proof is routine; we just show item 2: indeed, y G5 and G4, $x x^{-1} x=$ $u(d(x)) x=x$. The second identity is shown analogously.

Every set groupoid $\mathcal{G}$ can be naturally associated with a unital involutive quantale $\mathcal{P}(\mathcal{G})$, based on the complete join-semilattice $\left(\mathcal{P}\left(G_{1}\right), \cup\right.$ ) (see [12] and [13, Section 1.1] for a more detailed discussion): indeed, the product and involution on $G_{1}$ can be lifted to $\mathcal{P}\left(G_{1}\right)$ as follows:

$$
S \cdot T=\{x \cdot y \mid x \in S, y \in T \text { and } r(x)=d(y)\} \quad S^{*}=\left\{x^{-1} \mid x \in S\right\} .
$$

Let $E$ be the image of the structure map $u: G_{0} \rightarrow G_{1}$.

Definition 2.6 For every set groupoid $\mathcal{G}$, let $\mathcal{P}(\mathcal{G})=\left(\mathcal{P}\left(G_{1}\right), \cup, \cdot,()^{*}, E\right)$ be its associated discrete groupoid quantale.

Fact 2.7 $\mathcal{P}(\mathcal{G})$ is an $S G$-quantale for every set groupoid $\mathcal{G}$.

Proof The proof is routine: for instance let us show that $S \cdot E=S=E \cdot S$ for every $S \in \mathcal{P}\left(G_{1}\right)$ : indeed, one inclusion immediately follows from G4, and conversely if $x \cdot u(p)$ is defined, then, by $\mathrm{G} 2$, we have that $r(x)=d(u(p))=p$, and again by $\mathrm{G} 4$, we get $x \cdot u(p)=x \cdot u(r(x))=x$.

The axiom SG follows from Lemma 2.5.2.

\section{Spatial SGF Quantales and their Representation}

In [8], spatial SGF quantales are axiomatically introduced and proved to be representable as sub involutive quantales of $\mathcal{P}(\mathcal{G})$ for some groupoid $\mathcal{G}$. In this section, we are going to report on these results, referring to [8] for the proofs.

Definition 3.1 (cf. Definition 4.1 in [8]) A strongly Gelfand and functionallygenerated quantale (abbreviated as $S G F$ quantale) is a unital involutive quantale $\mathcal{Q}$ satisfying the following extra axioms:

SGF1. $\mathcal{Q}$ is $\bigvee$-generated by $\mathcal{I}(\mathcal{Q})$.

SGF2. $f=f f^{*} f$ for every $f \in \mathcal{I}(\mathcal{Q})$.

SGF3. For any $f, g \in \mathcal{I}(\mathcal{Q})$ and $h \in \mathcal{Q}_{e}$ if $f \leq h \cdot 1 \vee g$ then $f \leq h \cdot f \vee g$.

Axioms SGF1-2 readily imply that every SGF-quantale is SG. Let $\mathcal{Q}$ be an SGFquantale. For every $f \in \mathcal{I}(\mathcal{Q})$ let $d(f)=f f^{*}$ and $r(f)=f^{*} f$. Let $\mathcal{P}_{e}$ be the set of the prime elements of $\mathcal{Q}_{e}$ (cf. [5]) i.e. those non-top elements $p \in \mathcal{Q}_{e}$ s.t. for every $h, k \in \mathcal{Q}_{e}$, if $h \wedge k \leq p$, then $h \leq p$ or $k \leq p$. Let

$$
\mathcal{I}=\left\{(p, f) \in \mathcal{P}_{e} \times \mathcal{I}(\mathcal{Q}) \mid p \in \mathcal{P}_{e}, d(f) \not \leq p\right\} .
$$

Informally, the prime elements of $\mathcal{Q}_{e}$ can be thought of as the points (or rather, as the closures of points) of a topological space of which $\mathcal{Q}_{e}$ is the topology; elements $f \in \mathcal{I}(\mathcal{Q})$ can be thought of as partial homeomorphisms $f: d(f) \rightarrow r(f)$ between open sets of this space. Under this interpretation, the element $e \in \mathcal{I}(\mathcal{Q})$ is the identity homeomorphism, any $h \in \mathcal{Q}_{e}$ is both an open set of the space and the restriction of 
the identity homeomorphism to the open set $h$, and $f^{*}: r(f) \rightarrow d(f)$ is the inverse of $f$. Moreover, the inequality $d(f) \not \leq p$ means that the point $p$ belongs to the domain of $f$, hence the set $\mathcal{I}$ is essentially the space in which the operation ' $f$ applied to $p$ ' is defined. Lemma 4.7 and Proposition 4.8 in [8] describe the behaviour of these definitions, which, under the interpretation given above, is just the expected one. For instance, (cf. [8], Lemma 4.7.1) for every $(p, f) \in \mathcal{I}$, there exists a unique element $q \in$ $\mathcal{P}_{e}$ s.t. $r(f) \not \leq q$ and $p f=f q$. This prime element is denoted by $f[p]$, and represents the point ' $f(p)$ ' (or rather, the $f$-direct image of the closure of $p$ ). The following definition is meant to express, in the language of SGF-quantales, that ' $f(p)=g(p)$ ' for two such given partial homeomorphisms (or rather, that $f$ and $g$ coincide over the intersection of some open neighbourhood $h$ of $p$ with the closure of $p$ ):

Definition 3.2 (cf. Definition 4.5 in [8]) The incidence relation $\sim$ on $\mathcal{I}$ is defined by setting

$$
(p, f) \sim(q, g) \text { iff } p=q \text { and } h \not \leq p \text { and } h f \leq p f \vee g \text { for some } h \leq d(f) \wedge d(g) .
$$

We will also alternatively write $f \sim_{p} g$ (read: $f$ and $g$ are incident in $p$ ) in place of $(p, f) \sim(q, g)$.

The relation $\sim$ is an equivalence relation (cf. Proposition 4.6.1 in [8]), so we can consider the quotient set $\mathcal{I} / \sim$. Elements in $\mathcal{I} / \sim$ will be denoted by $[p, f],[q, g]$, etc.

Definition 3.3 (cf. Definition 4.9 in [8]) For every SGF-quantale $\mathcal{Q}$, its associated set groupoid $\mathcal{G}(\mathcal{Q})$ is defined as follows (see discussion above for notation): $G_{0}=\mathcal{P}_{e}$ and $G_{1}=\mathcal{I} / \sim$; moreover, the structure maps of $\mathcal{G}(\mathcal{Q})$ are given by the following assignments:

$$
\begin{gathered}
d([p, f])=p, \quad r([p, f])=f[p], \quad u(p)=[p, e], \\
{[p, f][q, g]=[p, f g] \quad \text { only if } \quad q=f[p]} \\
{[p, f]^{-1}=\left[f[p], f^{*}\right] .}
\end{gathered}
$$

For every SGF-quantale $\mathcal{Q}$, the structure $\mathcal{G}(\mathcal{Q})$ is indeed a set groupoid (cf. Proposition 4.11 in [8]). The SGF axiomatization of Definition 3.1 is constructive, i.e., it does not imply the 'existence of enough points'; its point-set strengthening is given below:

Definition 3.4 (cf. Definition 5.1 in [8]) For every SGF-quantale $\mathcal{Q}$ and every $[p, f] \in \mathcal{I} / \sim$, let

$$
\mathcal{I}_{[p, f]}=\{g \in \mathcal{I}(\mathcal{Q}) \mid d(g) \leq p \text { or }(p, g) \nsim(p, f)\} \quad \text { and } \quad I_{[p, f]}=\bigvee \mathcal{I}_{[p, f]} .
$$

$\mathcal{Q}$ is spatial if:

SPQ1. $f \not \leq I_{[p, f]}$ for every $(p, f) \in \mathcal{I}$.

SPQ2. $a=\bigwedge\left\{I_{[p, f]} \mid a \leq I_{[p, f]}\right\}$ for every $a \in \mathcal{Q}$. 
3.1 The Canonical Embedding

For every SGF-quantale $\mathcal{Q}$, let $\mathcal{P}(\mathcal{G}(\mathcal{Q}))$ ) be defined according to Definitions 3.3 and 2.6, and let $\alpha: \mathcal{Q} \rightarrow \mathcal{P}(\mathcal{G}(\mathcal{Q})))$ be defined by

$$
\alpha(a)=\left\{[p, f] \mid a \not \leq I_{[p, f]}\right\} .
$$

Theorem 3.5 (cf. Theorem 6.2 in [8]) For every $S G F$-quantale $\mathcal{Q}$,

1. $\alpha\left(\bigvee_{i} a_{i}\right)=\bigcup_{i} \alpha\left(a_{i}\right)$ for any family $\left\{a_{i} \mid i \in I\right\}$ of elements of $\mathcal{Q}$.

2. if $\mathcal{Q}$ is spatial, then $\alpha$ is an embedding.

3. $\alpha(a b)=\alpha(a) \alpha(b)$ for any $a, b \in \mathcal{Q}$.

4. $\alpha\left(a^{*}\right)=\alpha(a)^{*}$ for any $a \in \mathcal{Q}$.

5. $\alpha(1)=G_{1}$ and $\alpha(e)=u\left[G_{0}\right]$.

So, if $\mathcal{Q}$ is spatial, then $\alpha$ is a strong and strictly unital embedding of unital involutive quantales.

\section{Representation Theory}

As mentioned early on, the map $\alpha$ in Theorem 3.5 above plays the same crucial role as the embedding of a relation algebra into its perfect extension plays in [3]. The reason why this is important is that perfect extensions enjoy the additional property of being atomistic, i.e., join generated by their atoms. In the present paper, $F A$-quantales (Definition 4.1 below) are the quantale counterparts of atomistic relation algebras. In the two following subsections, we develop the theory of FA-quantales, show that they are identifiable with the quantales $\mathcal{P}(\mathcal{G})$ for any groupoid $\mathcal{G}$ (cf. Proposition 4.6), and provide a dual characterization of homomorphisms of FA-quantales and embeddings of FA-quantales which preserve finite meets (cf. Propositions 4.7, 4.9 and 4.10).

\subsection{FA-quantales and Set Groupoids}

For every quantale $\mathcal{Q}$, let $\operatorname{At}(\mathcal{Q})$ denote the collection of the atoms ${ }^{5}$ of $\mathcal{Q}$.

Definition 4.1 A functionally atomic quantale (abbreviated as FA-quantale) is an SG-quantale $\mathcal{L}$ s.t. its underlying complete join-semilattice is a complete atomic boolean algebra, and

FA. $A t(\mathcal{L}) \subseteq \mathcal{F}(\mathcal{L})$.

It immediately follows from the definition that $a=a a^{*} a$ for every $a \in A t(\mathcal{L})$. For every quantale $\mathcal{Q}$, if $a \in A t(\mathcal{Q})$, then $a^{*} \in A t(\mathcal{Q})$ : indeed, the following chain $0^{*}=$ $0 \neq b \leq a^{*}$ implies that $0^{* *}=0 \neq b^{*} \leq a^{* *}=a$, which implies that $b^{*}=0$ (hence $b=0$ ), or $b^{*}=a$ (hence $\left.b=a^{*}\right)$. This immediately implies that $\operatorname{At}(\mathcal{L}) \subseteq \mathcal{I}(\mathcal{L})$ for every FA-quantale $\mathcal{L}$. For every $c \in \mathcal{L}$, let $\operatorname{At}(c)=\{a \in A t(\mathcal{L}) \mid a \leq c\}$.

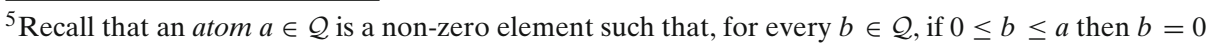
or $b=a$. 
Proposition 4.2 For every groupoid $\mathcal{G}$, the quantale $\mathcal{P}(\mathcal{G})$ is an FA-quantale.

Proof By Fact 2.7, the quantale $\mathcal{P}(\mathcal{G})$ is an SG-quantale, and its underlying complete join-semilattice is clearly a complete atomic boolean algebra. Atoms $a \in \mathcal{P}(\mathcal{G})$ are of the form $\{x\}$ for $x \in G_{1}$. By Definition 2.6 and G5, we have that $a^{*} a=\{x\}^{*} \cdot\{x\}=$ $\left\{x^{-1}\right\} \cdot\{x\}=\left\{x^{-1} x\right\}=\{u(r(x))\} \subseteq u\left[G_{0}\right]=e_{\mathcal{P}(\mathcal{G})}$.

Hence, the quantale $\mathcal{P}(\mathcal{G}(\mathcal{Q})$ ) is an FA-quantale for every SGF-quantale $\mathcal{Q}$.

Proposition 4.3 For every FA-quantale $\mathcal{L}$ and every $a \in A t(\mathcal{L})$,

1. if $f \in \mathcal{I}(\mathcal{L})$, then either af $=0$ or af $\in \operatorname{At}(\mathcal{L})$;

2. if $b \in \operatorname{At}(\mathcal{L})$, then either $a b=0$ or $a b \in \operatorname{At}(\mathcal{L})$;

3. $a a^{*}, a^{*} a \in \operatorname{At}(\mathcal{L})$;

4. $A t\left(\mathcal{L}_{e}\right)=\left\{a a^{*} \mid a \in \operatorname{At}(\mathcal{L})\right\}$.

Proof

1. If $a f \neq 0$, then there exists some $b \in A t(a f)$ such that $b f^{*} \neq 0$ : indeed, otherwise af $f^{*}=\bigvee\left\{c f^{*} \mid c \in A t(a f)\right\}=0$ and so $a f=a f f^{*} f=0$. Hence we get that $0 \neq$ $b f^{*} \leq a f f^{*} \leq a$; since $a \in A t(\mathcal{L})$, this implies that $b f^{*}=a$; hence we get that $0 \neq a f=b f^{*} f \leq b \in A t(a f)$, and so we conclude that $a f=b \in \operatorname{At}(\mathcal{L})$.

2. Immediate from item 1 and the observation that $\operatorname{At}(\mathcal{L}) \subseteq \mathcal{I}(\mathcal{L})$.

3. Immediate from item 2 and the observation that either $a^{*} a=0$ or $a a^{*}=0$ imply that $a=a a^{*} a=0$.

4. The right-to-left inclusion immediately follows from item 3 , the assumption FA, and $\operatorname{At}\left(\mathcal{L}_{e}\right)=\operatorname{At}(\mathcal{L}) \cap \mathcal{L}_{e}$. The converse direction follows from the latter identity and the fact that, by Proposition 2.2.2, it follows that $a a^{*}=a$ for every $a \in \mathcal{L}_{e}$.

Since the complete join-semilattice reduct of any FA-quantale is a complete atomic boolean algebra, the meet in FA-quantales is frame-distributive. ${ }^{6}$ By framedistributivity, it is easy to show that, for any FA-quantale $\mathcal{L}$, the atoms of $\mathcal{L}$, being completely join-irreducible, are completely join-prime. ${ }^{7}$ This implies for instance that

Lemma 4.4 For every FA-quantale $\mathcal{L}$, and all $x, y \in \mathcal{L}$,

1. $A t(x y)=\left\{a^{\prime} b^{\prime} \mid a^{\prime} b^{\prime} \neq 0, a^{\prime} \in \operatorname{At}(x)\right.$ and $\left.b^{\prime} \in \operatorname{At}(y)\right\}$;

2. $\operatorname{At}\left(x^{*}\right)=\left\{a^{*} \mid a \in \operatorname{At}(x)\right\}$.

Proof (1) By Proposition 4.3.2, if $a^{\prime} \in A t(x), b^{\prime} \in A t(y)$ and $a^{\prime} b^{\prime} \neq 0$, then $a^{\prime} b^{\prime} \in$ $A t(x y)$. As to the left-to-right inclusion, if $c \in A t(\mathcal{L})$, then $c$ is completely joinprime; so $c \leq x y=\bigvee A t(x) \bigvee A t(y)=\bigvee\left\{a^{\prime} b^{\prime} \mid a^{\prime} b^{\prime} \neq 0, a^{\prime} \in A t(x)\right.$ and $\left.b^{\prime} \in \operatorname{At}(y)\right\}$ implies that $0 \neq c \leq a^{\prime} b^{\prime}$ for some $a^{\prime} \in A t(x)$ and $b^{\prime} \in A t(y)$. Since $a^{\prime} b^{\prime}$ is an atom by Proposition 4.3.2, we conclude that $c=a^{\prime} b^{\prime}$. The proof of (2.) is similar.

\footnotetext{
${ }^{6}$ In fact, every FA-quantale is an inverse quantal frame (cf. [13]); moreover, since inverse quantal frames are SGF-quantales (cf. [8]), FA-quantales form a special subclass of SGF-quantales.

${ }^{7}$ A non-bottom element $c \in \mathcal{Q}$ is completely join-irreducible if, for every $S \subseteq \mathcal{Q}$, if $c=\bigvee S$ then $c \in S$; such a $c$ is completely join-prime if, for every $S \subseteq \mathcal{Q}$, if $c \leq \bigvee S$ then $c \leq s$ for some $s \in S$.
} 
Definition 4.5 For every FA-quantale $\mathcal{L}$, let

$$
\mathcal{G}(\mathcal{L})=\left(\operatorname{At}\left(\mathcal{L}_{e}\right), \operatorname{At}(\mathcal{L}), \cdot, d, r, u,()^{-1}\right),
$$

where $d(a)=a a^{*}$ and $r(a)=a^{*} a$, the map $u$ is the inclusion $\operatorname{At}\left(\mathcal{L}_{e}\right) \subseteq \operatorname{At}(\mathcal{L})$, the involution is given by $a^{-1}:=a^{*}$ and is the restriction of the quantale product, wherever it is non zero.

Proposition 4.6 For every FA-quantale $\mathcal{L}$, and every groupoid $\mathcal{G}$,

1. The structure $\mathcal{G}(\mathcal{L})$ is a groupoid, and the map $c \mapsto A t(c)$ defines a isomorphism $\mathcal{L} \cong \mathcal{P}(\mathcal{G}(\mathcal{L}))$.

2. The map $x \mapsto\{x\}$ defines an isomorphism $\mathcal{G} \rightarrow \mathcal{G}(\mathcal{P}(\mathcal{G})$ ).

\section{Proof}

1. The proof that Axioms G1-5 hold is straightforward. By atomicity of $\mathcal{L}$, joinprimeness of the atoms, and Lemma 4.4, it is not difficult to show that the assignments $c \mapsto A t(c)$ for every $c \in \mathcal{L}$ and $S \mapsto \bigvee S$ for every $S \subseteq A t(\mathcal{L})$ are inverse to one another and define strictly unital homomorphisms of unital involutive quantales.

2. Immediate.

\subsection{Dual Characterization of Strictly Unital Homomorphisms of FA-quantales}

The correspondence between FA-quantales and set groupoids given in the subsection above can be extended and framed in the context of a duality between the appropriate categories. In this section, we do not cover it in detail, and we limit ourselves to presenting those of its components which are relevant to the purposes of the present paper; the most important of these components is the dual characterization of the strictly unital homomorphisms of FA-quantales.

For any completely join-preserving map $h: \mathcal{L} \rightarrow \mathcal{L}^{\prime}$, define the relation $R_{h} \subseteq$ $A t\left(\mathcal{L}^{\prime}\right) \times A t(\mathcal{L})$ by setting $a^{\prime} R_{h} a$ iff $a^{\prime} \leq h(a)$, and let $R_{h}^{-1}[a]:=\left\{a^{\prime} \mid a^{\prime} R_{h} a\right\} .{ }^{8}$

Proposition 4.7 The following are equivalent for any completely join-preserving map $h: \mathcal{L} \rightarrow \mathcal{L}^{\prime}:$

1. the map $h$ is a strictly unital homomorphism of FA-quantales;

2. the relation $R_{h}$ satisfies the following properties for all $a, b \in \operatorname{At}(\mathcal{L})$ :

$$
\begin{gathered}
\operatorname{At}\left(\mathcal{L}_{e}^{\prime}\right)=\bigcup\left\{R_{h}^{-1}[a] \mid a \in \operatorname{At}\left(\mathcal{L}_{e}\right)\right\}, \quad R_{h}^{-1}\left[a^{*}\right]=\left\{a^{\prime *} \mid a^{\prime} \in R_{h}^{-1}[a]\right\}, \\
R_{h}^{-1}[a b]=\left\{a^{\prime} b^{\prime} \mid a^{\prime} b^{\prime} \neq 0, a^{\prime} \in R_{h}^{-1}[a] \text { and } b^{\prime} \in R_{h}^{-1}[b]\right\} .
\end{gathered}
$$

\footnotetext{
${ }^{8}$ This notation is standard, and is unrelated to the notions and notation of Section 3 .
} 
Proof The fact that $h$ is completely join-preserving implies that $h$ preserves products iff $h$ preserves products restricted to atoms. Indeed, assuming the latter, we have, for all $x, y \in \mathcal{L}$ :

$$
\begin{aligned}
h(x) h(y) & =\bigvee\{h(a) h(b) \mid a \in \operatorname{At}(x), b \in \operatorname{At}(y)\} \\
& =\bigvee\{h(a b) \mid a b \neq 0, a \in \operatorname{At}(x), b \in \operatorname{At}(y)\} \\
& =h(\bigvee\{a b \mid a b \neq 0, a \in \operatorname{At}(x), b \in \operatorname{At}(y)\}) \\
& =h(\bigvee \operatorname{At}(x y))=h(x y) . \quad(\text { Lemma 4.4.1) }
\end{aligned}
$$

Then by atomicity, for all $a, b \in A t(\mathcal{L})$,

$$
\begin{aligned}
& h(a b)=h(a) h(b) \\
& \text { iff } \quad \forall c \in \operatorname{At}\left(\mathcal{L}^{\prime}\right)[c \leq h(a b) \Leftrightarrow c \leq h(a) h(b)] \\
& \text { iff } \quad \forall c \in A t\left(\mathcal{L}^{\prime}\right)\left[c \in R_{h}^{-1}[a b] \Leftrightarrow c=a^{\prime} b^{\prime} \text { for some } a^{\prime} \leq h(a), b^{\prime} \leq h(b)\right] \\
& \text { iff } \quad R_{h}^{-1}[a b]=\left\{a^{\prime} b^{\prime} \mid a^{\prime} b^{\prime} \neq 0, a^{\prime} \in R_{h}^{-1}[a] \text { and } b^{\prime} \in R_{h}^{-1}[b]\right\} .
\end{aligned}
$$

Likewise, using Lemma 4.4.2, one shows that $h$ preserves involution iff $h$ preserves involution restricted to atoms. Then by atomicity, for every $a \in \operatorname{At}(\mathcal{L})$,

$$
\begin{array}{ll} 
& h\left(a^{*}\right)=h(a)^{*} \\
\text { iff } \quad \forall c \in A t\left(\mathcal{L}^{\prime}\right)\left[c \leq h\left(a^{*}\right) \Leftrightarrow c \leq h(a)^{*}\right] \\
\text { iff } \quad \forall c \in A t\left(\mathcal{L}^{\prime}\right)\left[c \in R_{h}^{-1}\left[a^{*}\right] \Leftrightarrow c=a^{\prime *} \text { for some } a^{\prime} \leq h(a)\right] \\
\text { iff } \quad R_{h}^{-1}\left[a^{*}\right]=\left\{a^{\prime *} \mid a^{\prime} \in R_{h}^{-1}[a]\right\} .
\end{array}
$$

Finally, by atomicity,

$$
\begin{array}{cl}
e^{\prime}=h(e) \quad \text { iff } \quad \forall c \in A t\left(\mathcal{L}^{\prime}\right)\left[c \leq e^{\prime} \Leftrightarrow c \leq h(e)=\bigvee\left\{h(a) \mid a \in \operatorname{At}\left(\mathcal{L}_{e}\right)\right\}\right] \\
\text { iff } \quad \forall c \in \operatorname{At}\left(\mathcal{L}^{\prime}\right)\left[c \in \operatorname{At}\left(\mathcal{L}_{e}^{\prime}\right) \Leftrightarrow c \in \bigcup\left\{R_{h}^{-1}[a] \mid a \in \operatorname{At}\left(\mathcal{L}_{e}\right)\right\}\right] \\
\text { iff } \quad \operatorname{At}\left(\mathcal{L}_{e}^{\prime}\right)=\bigcup\left\{R_{h}^{-1}[a] \mid a \in \operatorname{At}\left(\mathcal{L}_{e}\right)\right\} .
\end{array}
$$

Conversely, if $\mathcal{G}^{\prime}$ and $\mathcal{G}$ are groupoids, and $R \subseteq G_{1}^{\prime} \times G_{1}$, then the assignment $c \mapsto \bigvee \bigcup\left\{R^{-1}[a] \mid a \in A t(c)\right\}$ defines a completely join-preserving map $h_{R}: \mathcal{P}(\mathcal{G}) \rightarrow$ $\mathcal{P}\left(\mathcal{G}^{\prime}\right)$.

The following proposition essentially says that the obvious defining assignments for the unit and the counit of the dual equivalence mentioned at the beginning of the present subsection yield indeed natural transformations, in the appropriate categories: 
Proposition 4.8 For every completely join-preserving map $h: \mathcal{L} \rightarrow \mathcal{L}^{\prime}$, and every $R \subseteq$ $G_{1}^{\prime} \times G_{1}$,

1. the isomorphisms $\mathcal{L} \cong \mathcal{P}(\mathcal{G}(\mathcal{L}))$ and $\mathcal{L}^{\prime} \cong \mathcal{P}\left(\mathcal{G}\left(\mathcal{L}^{\prime}\right)\right)$ of Proposition 4.6 .1 identify $h$ with $h_{R_{h}}$,

2. the isomorphisms $\mathcal{G} \cong \mathcal{G}(\mathcal{P}(\mathcal{G}))$ and $\mathcal{G}^{\prime} \cong \mathcal{G}\left(\mathcal{P}\left(\mathcal{G}^{\prime}\right)\right)$ of Proposition 4.6.2 identify the relation $R$ with $R_{h_{R}}$.

\section{Proof}

1. Modulo identifying $\mathcal{P}\left(\mathcal{G}(\mathcal{L})\right.$ ) with $\mathcal{L}$ and $\mathcal{P}\left(\mathcal{G}\left(\mathcal{L}^{\prime}\right)\right.$ ) with $\mathcal{L}^{\prime}$ (via the identification of $\{x\}$ with $x$, cf. Proposition 4.6.2),

$$
\begin{aligned}
h_{R_{h}}(c) & =\bigvee \bigcup\left\{R_{h}^{-1}[x] \mid x \in \operatorname{At}(c)\right\} \\
& =\bigvee\left\{\bigvee R_{h}^{-1}[x] \mid x \in \operatorname{At}(c)\right\} \\
& =\bigvee\{h(x) \mid x \in \operatorname{At}(c)\}=h(c) .
\end{aligned}
$$

2. Notice that if $c \in A t(\mathcal{L})$, then $h_{R}(c)=R^{-1}[c]$. Modulo identifying $\mathcal{G}\left(\mathcal{P}\left(\mathcal{G}^{\prime}\right)\right)$ with $\mathcal{G}^{\prime}$ and $\mathcal{G}(\mathcal{P}(\mathcal{G})$ ) with $\mathcal{G}$ as before, we have:

$$
x^{\prime} R_{h_{R}} x \text { iff }\left\{x^{\prime}\right\} \subseteq h_{R}(\{x\})=R^{-1}[x] \text { iff } x^{\prime} R x .
$$

As a consequence of Propositions 4.7 and 4.8, we get:

Proposition 4.9 The following are equivalent for $R \subseteq G_{1}^{\prime} \times G_{1}$ :

1. $R \subseteq G_{1}^{\prime} \times G_{1}$ satisfies the following properties:

R1. $u^{\prime}\left[G_{0}^{\prime}\right]=\bigcup\left\{R^{-1}[u(p)] \mid p \in G_{0}\right\}$,

R2. $R^{-1}[i(x)]=\left\{i^{\prime}\left(x^{\prime}\right) \mid x^{\prime} \in R^{-1}[x]\right\}$,

R3. $R^{-1}[x y]=\left\{x^{\prime} y^{\prime} \mid x^{\prime} \in R^{-1}[x]\right.$ and $\left.y^{\prime} \in R^{-1}[y]\right\}$;

2. $h_{R}: \mathcal{P}(\mathcal{G}) \rightarrow \mathcal{P}\left(\mathcal{G}^{\prime}\right)$ is a strictly unital homomorphism of FA-quantales.

Proposition 4.10 The following are equivalent for $R \subseteq G_{1}^{\prime} \times G_{1}$ :

1. $R$ is the graph of a partial function onto $G_{1}$, i.e. $\pi_{2}[R]=G_{1}$, and if $a^{\prime} R b$ and $a^{\prime} R c$ then $b=c$;

2. $h_{R}: \mathcal{P}(\mathcal{G}) \rightarrow \mathcal{P}\left(\mathcal{G}^{\prime}\right)$ is an order embedding which preserves finite meets.

Proof In order to prove (2.) from (1.), it is enough to show that $h_{R}(x) \subseteq h_{R}(y)$ implies that $x \leq y$ for $x, y \in \mathcal{P}(\mathcal{G})$. By atomicity, it is enough to show that $\operatorname{At}(x) \subseteq$ $A t(y)$. If $c \in A t(x)$, then there exists some $a^{\prime} \in G_{1}^{\prime}$ such that $a^{\prime} \in R^{-1}[c]$. The assumption $h_{R}(x) \subseteq h_{R}(y)$ implies that $a^{\prime} \in R^{-1}[b]$ for some $b \in A t(y)$. Then, by the second part of assumption (1.), we conclude that $c=b \in A t(y)$. The remaining bit follows from the observation that inverse images of functions preserve intersections. In order 
to prove (1.) from (2.), by injectivity we get that $R^{-1}[a]=h_{R}(a) \neq 0$ for every atom $a$, which proves that $R$ is onto. If $a^{\prime} R b$ and $a^{\prime} R c$, then $a^{\prime} \in R^{-1}[b] \cap R^{-1}[c]$, hence we have that $0 \neq h_{R}(b) \wedge h_{R}(c)=h_{R}(b \wedge c)$. By injectivity, this implies that $b \wedge c \neq 0$, and since $b$ and $c$ are atoms, this implies that $b=c$.

Remark 4.11 Although this is not immediately relevant to the purposes of the present paper, it is worth mentioning that the category-theoretic perspective outlined in the present subsection can be further extended from a dual equivalence between appropriate categories of FA-quantales and set-groupoids to a dual adjunction between appropriate categories of SGF-quantales and set groupoids; this is due to the fact that, as we mentioned early on at the beginning of Section 4.1, FA-quantales form a subclass of SGF-quantales. The dual adjunction is defined on objects by the assignments $\mathcal{Q} \mapsto \mathcal{G}(\mathcal{Q})$ (cf. Definition 3.3) for every SGF-quantale $\mathcal{Q}$, and $\mathcal{G} \mapsto \mathcal{P}(\mathcal{G})$ for every set-groupoid $\mathcal{G}$. The counit of this adjunction is given by the canonical embedding $\alpha: \mathcal{Q} \mapsto \mathcal{P}(\mathcal{G}(\mathcal{Q}))$ of Theorem 3.5.

\subsection{Relational Representations for Spatial SGF-quantales}

The dual characterization of strictly unital homomorphisms of FA-quantales of the previous subsection can be used to obtain an optimal relational representation for spatial SGF-quantales (cf. Corollary 4.15).

Indeed, let $\mathcal{L}$ be an FA-quantale, let $\mathcal{G}=\mathcal{G}(\mathcal{L})$, and $\mathcal{G}^{\prime}$ be the pair groupoid arising from $G_{1}=A t(\mathcal{L})$ (cf. Example 2.4.1); hence $G_{1}^{\prime}=G_{1} \times G_{1}$ and $G_{0}^{\prime}=G_{1}$. Consider the relation $R \subseteq G_{1}^{\prime} \times G_{1}$ such that $R=\operatorname{graph}(n)$, where $n: G_{1} \times G_{1} \rightarrow G_{1}$ is the partial map defined by $n(a, b)=a b^{*}$. Notice that, the SG condition implies that $c=$ $c c^{*} c=a b^{*}$ with $a=c$ and $b=c^{*} c$ for every $c \in A t(\mathcal{L})$, hence $n$ is onto $G_{1}$.

Then we have that $R^{-1}[c]=n^{-1}[c]=\left\{(a, b) \mid a b^{*}=c\right\}$ for every $c \in G_{1}$. Identifying $\mathcal{L}$ with $\mathcal{P}(\mathcal{G})$, by Proposition 4.10 , the completely join-preserving map $h_{R}: \mathcal{L} \rightarrow$ $\mathcal{P}\left(G_{1} \times G_{1}\right)$, defined by

$$
h_{R}(x)=\left\{R^{-1}[c] \mid c \in A t(x)\right\}=\left\{(a, b) \mid a, b \in A t(\mathcal{L}) \text { and } 0 \neq a b^{*} \leq x\right\},
$$

is an order embedding.

Lemma 4.12 for every $F A$-quantale $\mathcal{L}$, every $x \in \mathcal{L}$ and all $a, b \in \operatorname{At}(\mathcal{L})$,

$$
0 \neq a b^{*} \leq x \text { if and only if } a \leq x b .
$$

Proof Assume that $0 \neq a b^{*} \leq x$. By SG, $0 \neq a b^{*}=a b^{*} b b^{*}$, hence $a b^{*} b \neq 0$. Then, the element $b$ being functional implies that $0 \neq a b^{*} b \leq a$; since $a$ is an atom, this implies that $a=a b^{*} b \leq x b$. Conversely, if $a \leq x b$, then $a b^{*} \leq x b b^{*} \leq x$. Moreover, we have that $0 \neq a=a a^{*} a$ implies $0 \neq a a^{*} \leq x b a^{*}$, hence $x b a^{*} \neq 0$, and so $b a^{*} \neq 0$, i.e. $a b^{*} \neq 0$.

In view of the Lemma above, the embedding $h_{R}$ can be equivalently defined by

$$
h_{R}(x)=\{(a, b) \mid a, b \in A t(\mathcal{L}) \text { and } a \leq x b\} .
$$

Proposition 4.13 $h_{R}$ is a strictly unital quantale homomorphism. 
Proof By Proposition 4.9, it is enough to show that the conditions R1-3 hold for $R=\operatorname{graph}(n)$. As to R1, notice that Lemma 4.12 implies that for every $a, b \in \operatorname{At}(\mathcal{L})$,

$$
0 \neq a b^{*} \leq e \text { iff } a \leq b \text { iff } a=b .
$$

Hence,

$$
\begin{aligned}
\bigcup\left\{R^{-1}[u(p)] \mid p \in G_{0}\right\} & =\left\{(a, b) \mid a b^{*} \in \operatorname{At}\left(\mathcal{L}_{e}\right)\right\} \\
& =\{(a, a) \mid a \in \operatorname{At}(\mathcal{L})\}=u^{\prime}\left[G_{0}^{\prime}\right] .
\end{aligned}
$$

As to $\mathrm{R} 2$, recall that the structure map $i^{\prime}$ on pair groupoids is defined by $i^{\prime}(a, b)=$ $(a, b)^{-1}=(b, a)$; hence

$$
\begin{aligned}
R^{-1}[i(c)] & =R^{-1}\left[c^{*}\right]=\left\{(b, a) \mid b a^{*}=c^{*}\right\} \\
& =\left\{(a, b)^{-1} \mid a b^{*}=c\right\} \\
& =\left\{i^{\prime}\left(x^{\prime}\right) \mid x^{\prime} \in R^{-1}[c]\right\} .
\end{aligned}
$$

As to R3, recall that the product on pair groupoids is defined by $(a, b) \cdot\left(b^{\prime} c\right)=(a, c)$ if $b=b^{\prime}$; notice preliminarily that for all $a, b, c, d \in A t(\mathcal{L})$, if $c d \neq 0$ and $a b^{*}=$ $c d$, then $a b^{* *}=c$ and $b^{\prime} b^{*}=d$ for some $b^{\prime} \in A t(\mathcal{L})$ : indeed, by Lemma 4.12 , the chain $0 \neq a b^{*}=c d$ implies that $0 \neq a \leq c d b$, hence $a=c d b$. Hence we have that $0 \neq a b^{*}=c d b b^{*}$, which implies that $d b b^{*} \neq 0$. Since $b$ is a partial unit, we have $0 \neq d b b^{*} \leq d$, which implies $d b b^{*}=d$. Take $b^{\prime}=d b$. From $d b b^{*} \neq 0$, we get $b^{\prime}=$ $d b \neq 0$, so by Proposition 4.3.2, we conclude that $b^{\prime} \in A t(\mathcal{L})$. Since $a=c d b$, we have: $a b^{*}=(c d b)(d b)^{*}=c d b b^{*} d^{*} \leq c d d^{*} \leq c$. To finish the proof, it is enough to show that $a b^{*} \neq 0$. Indeed, the chain $0 \neq a=c d b=c d b(c d b)^{*} c d b=\left(c d b b^{*} d^{*}\right) c^{*} c d b=$ $a b^{* *} c^{*} c d b$ implies that $a b^{\prime *} \neq 0$. Therefore, the following chain of equalities holds:

$$
\begin{aligned}
R^{-1}[c d] & =\left\{(a, b) \mid a b^{*}=c d\right\} \\
& =\left\{\left(a, b^{\prime}\right)\left(b^{\prime}, b\right) \mid a b^{* *}=c \text { and } b^{\prime} b^{*}=d\right\} \\
& =\left\{x^{\prime} y^{\prime} \mid x^{\prime} \in R^{-1}[c] \text { and } y^{\prime} \in R^{-1}[d]\right\} .
\end{aligned}
$$

Summing up, we proved the following

Theorem 4.14 For every FA-quantale $\mathcal{L}$, the map $\mathcal{L} \rightarrow \mathcal{P}(A t(\mathcal{L}) \times A t(\mathcal{L}))$ defined by $x \mapsto F(x)=\{\langle a, b\rangle \mid a, b \in A t(\mathcal{L}), a \leq x b\}$ is a strictly unital embedding of unital involutive quantales.

Putting together Theorems 3.5 and 4.14, we obtain the quantale counterpart of Theorem 4.22 in [3]:

Corollary 4.15 Every spatial SGF-quantale is isomorphic to a sub unital involutive quantale of some quantale of relations.

Proof The composition of $\alpha$ of Theorem 3.5 and $F$ of Theorem 4.14 is a strictly unital embedding of unital involutive quantales. 
Notice that, since the embedding $\alpha$ of Theorem 3.5 does not need to preserve meets, neither does the embedding of the corollary above.

\subsection{Characterization of Quantales of Relations}

As a second application of the dual characterization of strictly unital quantale embeddings given in Section 4.2, in the present subsection, a characterization is given of those quantales which are isomorphic to $\mathcal{P}(R)$ for some equivalence relation $R$ (cf. Theorem 4.21).

We mentioned early on (cf. Example 2.4.1) that the groupoids arising from equivalence relations are characterized up to isomorphism as those groupoids $\mathcal{G}$ such that the product map $d \times r: G_{1} \rightarrow G_{0} \times G_{0}$ is injective. Now let $\mathcal{G}^{\prime}$ be the pair groupoid of $G_{0}$ (i.e., $G_{1}^{\prime}=G_{0} \times G_{0}$ ), and observe that $d \times r$ is injective if and only if the relation $R \subseteq\left(G_{0} \times G_{0}\right) \times G_{1}$, defined as the converse of $\operatorname{graph}(d \times r)$, is the graph of a partial map onto $G_{1}$, i.e., iff $R$ satisfies the conditions in item 1 of Proposition 4.10. By the same proposition, this is equivalent to $h_{R}: \mathcal{P}(\mathcal{G}) \rightarrow$ $\mathcal{P}\left(G_{0} \times G_{0}\right)$, given by

$$
\begin{aligned}
h_{R}(x) & =\bigcup\left\{R^{-1}[a] \mid a \in \operatorname{At}(x)\right\} \\
& =\{(d(a), r(a)) \mid a \in \operatorname{At}(x)\},
\end{aligned}
$$

being a completely join-preserving embedding which preserves finite meets.

Proposition 4.16 For every groupoid $\mathcal{G}$, the map $h_{R}$, where $R$ is the converse of graph $(d \times r)$, is a strictly unital quantale homomorphism.

Proof By Proposition 4.9, it is enough to show that the conditions R1-3 hold for $R$. As to R1, recall that, since $\mathcal{G}^{\prime}$ is the pair groupoid of $G_{0}$, we have that $G_{0}^{\prime}=G_{0}$ and $u^{\prime}\left[G_{0}^{\prime}\right]=\left\{(a, a) \mid a \in G_{0}\right\}$. Hence,

$$
\begin{aligned}
\bigcup\left\{R^{-1}[u(p)] \mid p \in G_{0}\right\} & =\left\{(d(u(p)), r(u(p))) \mid p \in G_{0}\right\} \\
& =\left\{(p, p) \mid p \in G_{0}\right\}=u^{\prime}\left[G_{0}^{\prime}\right] .
\end{aligned}
$$

As to $\mathrm{R} 2$, recall that the structure map $i^{\prime}$ on pair groupoids is defined by $i^{\prime}(a, b)=$ $(a, b)^{-1}=(b, a)$; hence

$$
\begin{aligned}
R^{-1}[i(x)] & =\{(d(i(x)), r(i(x)))\} \\
& =\{(r(x), d(x))\} \\
& =\left\{(d(x), r(x))^{-1}\right\} \\
& =\left\{i^{\prime}\left(x^{\prime}\right) \mid x^{\prime} \in R^{-1}[x]\right\} .
\end{aligned}
$$


As to R3, recall that if $x, y \in G_{1}$, the product $x y$ is defined iff $r(x)=d(y)$, and that the product on pair groupoids is defined by $(a, b) \cdot\left(b^{\prime} c\right)=(a, c)$ if $b=b^{\prime}$; therefore,

$$
\begin{aligned}
R^{-1}[x y] & =\{(d(x y), r(x y))\} \\
& =\{(d(x), r(y))\} \\
& =\{(d(x), r(x))(d(y), r(y))\} \\
& =\left\{x^{\prime} y^{\prime} \mid x^{\prime} \in R^{-1}[x] \text { and } y^{\prime} \in R^{-1}[y]\right\} .
\end{aligned}
$$

Notice that the proposition above was proven for an arbitrary groupoid $\mathcal{G}$, without the assumption that $d \times r$ be injective. Therefore, by Proposition 4.6.1, we have the following

Proposition 4.17 For every FA-quantale $\mathcal{L}$,

1. the map $\gamma: \mathcal{L} \rightarrow \mathcal{P}\left(\operatorname{At}\left(\mathcal{L}_{e}\right) \times \operatorname{At}\left(\mathcal{L}_{e}\right)\right)$, defined by

$$
\gamma(x)=\left\{\left(a a^{*}, a^{*} a\right) \mid a \in \operatorname{At}(x)\right\},
$$

is a strictly unital homomorphism of unital involutive quantales.

2. $\gamma$ is an embedding iff $\mathcal{L}$ is isomorphic to a quantale $\mathcal{P}(R)$ for some equivalence relation $R$.

Proof Item 1 follows from Proposition 4.16. The right-to-left direction of item 2 is easy to verify. Conversely, setting $R=\gamma(1)$, the inequalities $e \leq 1,1 \cdot 1 \leq 1$ and $1^{*}=$ 1 imply that $R$ is an equivalence relation. If $S \subseteq \gamma(1)$, then $S=\left\{\left(a a^{*}, a^{*} a\right) \mid a \in X\right\}$ for some $X \subseteq A t(\mathcal{Q})$. Then $\gamma(\bigvee X)=S$.

Lemma 4.18 The following are equivalent for any completely join-preserving map $g$ : $\mathcal{L} \rightarrow \mathcal{L}^{\prime}$ such that $g(c) \in \operatorname{At}\left(\mathcal{L}^{\prime}\right)$ fore every $c \in \operatorname{At}(\mathcal{L}):$

1. $g$ is an order embedding;

2. for all $a, b \in A t(\mathcal{L})$, if $g(a)=g(b)$ then $a=b$.

Proof If $g$ is an order embedding, then $g$ is injective. Conversely, if $x, y \in \mathcal{L}$ and $g(x) \leq g(y)$, then, by the atomicity of $\mathcal{L}$, to show that $x \leq y$, it is enough to show that $A t(x) \subseteq A t(y)$. If $a \in A t(x)$, then $g(a) \in A t(g(x)) \subseteq A t(g(y))$, so, since $g$ is completely join-preserving, we get that $g(a) \leq g(y)=\bigvee\{g(b) \mid b \in A t(y)\}$. Since $g(a)$ is completely join-prime, this implies that $0 \neq g(a) \leq g(b)$ for some $b \in A t(y)$, which implies, since $g(b)$ is an atom, that $g(a)=g(b)$. Hence we conclude that $a=b \in A t(y)$.

For every FA-quantale $\mathcal{L}$ and every $c \in A t(\mathcal{L})$, let

$$
\operatorname{Stab}(\mathcal{L})=\left\{c \in \operatorname{At}(\mathcal{L}) \mid c c^{*}=c^{*} c\right\} .
$$


Remark 4.19 The notation $\operatorname{Stab}(\mathcal{L})$ is motivated by the existence of a partially defined action $\operatorname{At}(\mathcal{L}) \times \operatorname{At}\left(\mathcal{L}_{e}\right) \rightarrow \operatorname{At}\left(\mathcal{L}_{e}\right)$ given by $(c, a) \mapsto c(a)=c^{*} a c$. One can show that $c(a)=a$ if and only if $h=c c^{*}=c^{*} c$. Hence $\operatorname{Stab}(\mathcal{L})$ can be regarded as the set of atoms stabilizing some atom in $\mathcal{L}_{e}$.

Lemma 4.20 For every FA-quantale $\mathcal{L}$,

1. $\operatorname{Stab}(\mathcal{L})=\left\{a b a^{*} \mid a b a^{*} \neq 0\right.$ and $\left.a, b \in \operatorname{At}(\mathcal{L})\right\}$.

2. $a a^{*}=b b^{*}$ and $a^{*} a=b^{*} b$ iff $a b^{*}, a^{*} b \in \operatorname{Stab}(\mathcal{L})$.

\section{Proof}

1. If $a, b \in A t(\mathcal{L})$ and $c=a b a^{*} \neq 0$ then $c c^{*}=a b a^{*} a b^{*} a^{*} \leq a a^{*}$, hence $c c^{*}=a a^{*}$. Likewise we have $c^{*} c \leq a a^{*}$, which implies $c^{*} c=a a^{*}$. This shows that $c \in$ $\operatorname{Stab}(\mathcal{L})$. Conversely, if $c \in \operatorname{Stab}(\mathcal{L})$, then $0 \neq c=c c^{*} c=c c c^{*}$.

2. If $a a^{*}=b b^{*}$ and $a^{*} a=b^{*} b$, then $0 \neq a=a a^{*} a=a b^{*} b$, hence $a b^{*} \neq 0$. Therefore, by Proposition 4.3.2, we conclude that $a b^{*} \in A t(\mathcal{L})$. Hence, by Proposition 4.3.3, we have: $0 \neq\left(a b^{*}\right)\left(a b^{*}\right)^{*}=a b^{*} b a^{*} \leq a a^{*}$, which implies $\left(a b^{*}\right)\left(a b^{*}\right)^{*}=$ $a a^{*}$ and likewise $\left(a b^{*}\right)^{*}\left(a b^{*}\right)=b b^{*}$. Since $a a^{*}=b b^{*}$, we conclude that $a b^{*} \in \operatorname{Stab}(\mathcal{L})$. Conversely, suppose that $a b^{*} \in \operatorname{Stab}(\mathcal{L}) ;$ then $b b^{*} \geq b a^{*} a b^{*}=$ $\left(a b^{*}\right)^{*}\left(a b^{*}\right)=\left(a b^{*}\right)\left(a b^{*}\right)^{*}=a b^{*} b a^{*} \leq a a^{*}$ and by Proposition 4.3.3 this implies that $b b^{*}=a a^{*}$.

We are ready to give the quantale counterpart of Theorem 4.30 in [3]:

Theorem 4.21 The following are equivalent for every unital involutive quantale $\mathcal{Q}$ :

1. $\mathcal{Q}$ is isomorphic to a quantale $\mathcal{P}(R)$ for some equivalence relation $R$;

2. $\mathcal{Q}$ is an FA-quantale such that $a 1 a^{*} \in \operatorname{At}(\mathcal{Q})$ for every $a \in \operatorname{At}(\mathcal{Q})$.

Proof The '(1.) implies (2.)' direction is easy to check. For the converse, let $\mathcal{Q}=\mathcal{L}$ be an FA-quantale such that $a 1 a^{*} \in A t(\mathcal{L})$ for every $a \in A t(\mathcal{L})$. By Proposition 4.17 and Lemma 4.18, it is enough to show that the map $\gamma$, defined in Proposition 4.17, be injective over atoms. By Lemma 4.20.1, the assumptions imply that

$$
\operatorname{Stab}(\mathcal{L})=\left\{a b a^{*} \mid a b a^{*} \neq 0 \text { and } a, b \in \operatorname{At}(\mathcal{L})\right\}=\left\{a a^{*} \mid a \in \operatorname{At}(\mathcal{L})\right\}=\operatorname{At}\left(\mathcal{L}_{e}\right) .
$$

Hence, by Lemma 4.20.2, $\gamma(b)=\gamma(a)$ implies that $a b^{*} \leq e$, and so $a=a a^{*} a=$ $a b^{*} b \leq b$ which implies that $a=b$.

Open Access This article is distributed under the terms of the Creative Commons Attribution Noncommercial License which permits any noncommercial use, distribution, and reproduction in any medium, provided the original author(s) and source are credited.

\section{References}

1. Brown, C., Gurr, D.: A representation theorem for quantales. J. Pure Appl. Algebra 85(1), 27-42 (1993)

2. Gelfand, I.M., Naimark, M.A.: On the embedding of normed rings into the ring of operators in Hilbert space. Mat. Sb. 12, 197-213 (1943) 
3. Jónsson, B., Tarski, A.: Boolean algebras with operators, part II. Am. J. Math. 74(2), 127-162 (1952)

4. Kruml, D., Paseka, J.: Embeddings of quantales into simple quantales. J. Pure Appl. Algebra 148(2), 209-424 (2000)

5. Johnstone, P.T.: Stone Spaces. Cambridge Studies in Adv. Math., vol. 3. Cambridge University Press, Cambridge, UK (1982)

6. Mulvey, C.J.: \&. Rend. Circ. Mat. Palermo 12, 99-104 (1986)

7. Mulvey, C.J., Resende, P.: A noncommutative theory of Penrose tilings. Int. J. Theor. Phys. 44, $655-689(2005)$

8. Palmigiano, A., Re, R.: Groupoid quantales: a non-étale setting. J. Pure Appl. Algebra 215(8), 1945-1957 (2011)

9. Palmigiano, A., Re, R.: Topological Groupoid Quantales. In: Aerts, D., Smets, S., van Bendegem, J.P. (eds.) Special Issue: The Contributions of Logic to the Foundations of Physics. Studia Logica, vol. 95, pp. 125-137 (2010)

10. Paterson, A.L.T.: Groupoids, Inverse Semigroups, and their Operator Algebras. Progress in Mathematics, vol. 170. Birkhäuser, Boston (1999)

11. Protin, M.C., Resende, P.: Quantales of Open Groupoids. arXiv:0811.4539v2

12. Resende, P.: Lectures on Étale Groupoids, Inverse Semigroups and Quantales. Lecture Notes for the GAMAP IP Meeting, Antwerp (2006)

13. Resende, P.: Étale groupoids and their quantales. Adv. Math. 208, 147-209 (2007)

14. Rosenthal, K.: Quantales an their Applications. Pitman Research Notes in Math., vol. 234. Longman (1990)

15. Takesaki, M.: Theory of Operator Algebras I, EMS Series, vol. 124. Springer, New York (2002)

16. Valentini, S.: Representation Theorems for Quantales. Math. Log. Q. 40, 182-190 (1994) 